\title{
Coupling QCD-Scale Axionlike Particles to Gluons
}

\author{
Daniel Aloni, ${ }^{1, *}$ Yotam Soreq, ${ }^{2,3, \dagger}$ and Mike Williams ${ }^{4, \$}$ \\ ${ }^{1}$ Department of Particle Physics and Astrophysics, Weizmann Institute of Science, Rehovot, Israel 7610001 \\ ${ }^{2}$ Center for Theoretical Physics, Massachusetts Institute of Technology, Cambridge, Massachusetts 02139, USA \\ ${ }^{3}$ Theoretical Physics Department, CERN, CH-1211 Geneva 23, Switzerland \\ ${ }^{4}$ Laboratory for Nuclear Science, Massachusetts Institute of Technology, Cambridge, Massachusetts 02139, USA
}

(Received 6 December 2018; revised manuscript received 2 April 2019; published 19 July 2019)

\begin{abstract}
We present a novel data-driven method for determining the hadronic interaction strengths of axionlike particles (ALPs) with QCD-scale masses. Using our method, it is possible to calculate the hadronic production and decay rates of ALPs, along with many of the largest ALP decay rates to exclusive final states. To illustrate the impact on QCD-scale ALP phenomenology, we consider the scenario where the ALP-gluon coupling is dominant over the ALP coupling to photons, electroweak bosons, and all fermions for $m_{\pi} \lesssim m_{a} \lesssim 3 \mathrm{GeV}$. We emphasize, however, that our method can easily be generalized to any set of ALP couplings to standard model particles. Finally, using the approach developed here, we provide calculations for the branching fractions of $\eta_{c} \rightarrow V V$ decays; i.e., $\eta_{c}$ decays into two vector mesons, which are consistent with the known experimental values.
\end{abstract}

DOI: 10.1103/PhysRevLett.123.031803

Axionlike particles (ALPs) are hypothetical pseudoscalars whose couplings to the gauge bosons of the standard model (SM) - the gluons, photons, and electroweak bosons - are highly suppressed at low energies by a large cutoff scale $\Lambda$. ALPs are found in many proposed extensions to the SM (see Refs. [1-4]), since they naturally address such puzzles as the Strong $C P$ [5-8] and Hierarchy problems [9]. Moreover, ALPs may explain the long-standing anomaly with the magnetic moment of the muon [10], and could provide a portal connecting SM particles to dark matter [11-14].

ALPs are pseudo-Nambu-Goldstone bosons, and therefore, their masses, $m_{a}$, are expected to be $m_{a} \ll \Lambda$. Recently, MeV-to-GeV scale, henceforth QCD-scale, ALPs have received considerable interest [15-25]; however, the phenomenological impact of ALP-gluon interactions is not well understood for QCD-scale ALPs. The effective Lagrangian describing such interactions is

$$
\mathcal{L} \supset-\frac{4 \pi \alpha_{s} c_{g}}{\Lambda} a G^{\mu \nu} \tilde{G}_{\mu \nu},
$$

where $c_{g}$ is the dimensionless agg vertex coupling constant and $\tilde{G}_{\mu \nu} \equiv \frac{1}{2} \epsilon_{\mu \nu \alpha \beta} G^{\alpha \beta}$.

In this Letter, we present a novel data-driven method for determining the hadronic interaction strengths of

Published by the American Physical Society under the terms of the Creative Commons Attribution 4.0 International license. Further distribution of this work must maintain attribution to the author(s) and the published article's title, journal citation, and DOI. Funded by SCOAP ${ }^{3}$.
QCD-scale ALPs. Using our method, it is possible to calculate the hadronic production and decay rates of ALPs, along with many of the largest ALP decay branching fractions to exclusive final states. To illustrate the impact on QCD-scale ALP phenomenology of $c_{g} \neq 0$, we consider

$$
c_{g} \gg c_{\gamma}, c_{\mathrm{EW}}, c_{f},
$$

for $m_{\pi} \lesssim m_{a} \lesssim 3 \mathrm{GeV}$, i.e., the scenario where the ALPgluon coupling is dominant over the ALP coupling to photons $\left(c_{\gamma}\right)$, electroweak bosons $\left(c_{\mathrm{EW}}\right)$, and all fermions $\left(c_{f}\right)$. We emphasize, however, that our method can easily be generalized to any ALP couplings to SM particles. The impact of ALP couplings to photons, electroweak bosons, leptons, and heavy quarks is known [26], while additional direct couplings to light quarks are easily handled within our framework (see the Supplemental Material [27] to this Letter).

We begin by noting that ALP-lepton couplings arise at the three-loop order in this scenario, and therefore, are neglected throughout. ALP couplings to quarks are generated by the ALP-gluon interactions. Similarly, ALPphoton interactions are also generated by ALP-gluon interactions, though these are suppressed by $\mathcal{O}\left(\alpha_{\mathrm{EM}^{2}}{ }^{2}\right.$.

For low masses, ALP-gluon interactions can be studied using chiral perturbation theory $(\chi \mathrm{PT})$, while for $m_{a} \gg$ $\Lambda_{\mathrm{QCD}}$ perturbative QCD (PQCD) can be employed. However, no reliable calculations are available for most QCD-scale masses. Furthermore, PQCD only predicts the total hadronic decay rate. It does not inform experimenters which decays to look for, or how to determine the sensitivity of any exclusive decays. 
Since $a \rightarrow \pi \pi$ and $a \rightarrow \pi^{0} \gamma$ are forbidden by $C P$ and $C$, respectively, the dominant hadronic decays for low-mass ALPs will be $a \rightarrow 3 \pi^{0}$ and $a \rightarrow \pi^{+} \pi^{-} \pi^{0}$, even though they violate isospin, along with $a \rightarrow \pi^{+} \pi^{-} \gamma$, which is suppressed by a factor of $\alpha_{\mathrm{EM}}$ [28]. The decay rates are similar for both $3 \pi$ modes and to leading order (LO) in $\chi \mathrm{PT}$ are [26]

$\Gamma_{a \rightarrow 3 \pi} \approx \frac{\pi m_{a} m_{\pi}^{4} c_{g}^{2} \delta_{I}^{2}}{\Lambda^{2} f_{\pi}^{2}} \mathcal{K}_{3 \pi}\left(\frac{m_{\pi}^{2}}{m_{a}^{2}}\right)$ for $m_{a} \lesssim 1 \mathrm{GeV}$,

where $\delta_{I} \equiv\left(m_{d}-m_{u}\right) /\left(m_{d}+m_{u}\right) \approx 1 / 3$ is the isospin violation induced by $m_{u} \neq m_{d}$ and $\mathcal{K}_{3 \pi}$ contains the final-state kinematic factors (see Supplemental Material [27]). In the PQCD regime, the total rate to hadrons is $\Gamma_{a \rightarrow g g}$, which at one-loop order is [29]

$\Gamma_{a \rightarrow g g} \approx \frac{32 \pi \alpha_{s}^{2} c_{g}^{2} m_{a}^{3}}{\Lambda^{2}}\left(1+\frac{83 \alpha_{s}}{4 \pi}\right)$ for $m_{a} \gg \Lambda_{\mathrm{QCD}}$.

For $m_{a} \approx 2 \mathrm{GeV}$, the one-loop correction is comparable in size to the leading-order result, making this the smallest mass where Eq. (4) has $\mathcal{O}(1)$ validity. Naively, it is tempting to interpolate the total hadronic rate from where $a \rightarrow 3 \pi$ is the dominant hadronic decay to where the PQCD result is valid; however, even though such an interpolation only covers a factor of 4 in $m_{a}$, numerically

$$
\frac{\Gamma_{a \rightarrow g g}\left(m_{a}=2 \mathrm{GeV}\right)}{\Gamma_{a \rightarrow 3 \pi}\left(m_{a}=0.5 \mathrm{GeV}\right)} \approx \mathcal{O}\left(10^{5}\right) !
$$

Clearly a deeper understanding of the hadronic interactions of QCD-scale ALPs is required-which is our primary focus.

By performing a chiral transformation of the light-quark fields [30-32], we replace the $a g g$ vertex by ALP-quark axial-current couplings, which we subsequently match to the chiral Lagrangian. This leads to ALP- $\pi^{0}$ kinetic mixing and ALP- $\eta^{(/)}$kinetic and mass mixing making it possible to assign the ALP a $U(3)$ representation at low masses. We assign all ALPs up to $\approx 3 \mathrm{GeV}$ the $U(3)$ representation (Close to $3 \mathrm{GeV}$ mixing with the $\eta_{c}$ charmonium state should be considered. We leave this for future studies.)

$$
\frac{f_{\pi}}{f_{a}} \boldsymbol{a}=\frac{f_{\pi}}{f_{a}} \frac{\tilde{\alpha}_{s}\left(m_{a}\right)}{\sqrt{6}} \operatorname{diag}\left\{\mathcal{C}_{u}, \mathcal{C}_{d}, \mathcal{C}_{s}\right\},
$$

where $\mathcal{C}_{q}$ are $m_{a}$-dependent dimensionless constants, $f_{a} \equiv$ $-\Lambda / 32 \pi^{2} c_{g}$ is the ALP decay constant, and

$$
\tilde{\alpha}_{s}\left(m_{a}\right) \equiv \begin{cases}1 & \text { for } m_{a} \leq 1 \mathrm{GeV} \\ \alpha_{s}\left(m_{a}\right) & \text { for } m_{a}>1 \mathrm{GeV}\end{cases}
$$

accounts for $\alpha_{s}$ running which weakens ALP-gluon interactions at higher masses. (To obtain smooth results, we take $\alpha_{s}(1 \mathrm{GeV})=1$, then interpolate to the known value for $m_{a}>1.5 \mathrm{GeV}$.) Nota bene, we factored out $f_{\pi} / f_{a}$ to make this dependence explicit, and follow the normalization convention

$$
\langle\boldsymbol{P P}\rangle \equiv 2 \operatorname{Tr}[\boldsymbol{P P}]=1,
$$

for the pseudoscalar $U(3)$ generators $\boldsymbol{\pi}^{0}, \boldsymbol{\eta}$, and $\boldsymbol{\eta}^{\prime}$.

For $m_{a} \lesssim 1 \mathrm{GeV}$, we derive the ALP-P mixings, for $P=\pi^{0}, \eta, \eta^{\prime}$, using the $\mathrm{LO}$ chiral Lagrangian by extending previous works, e.g., Ref. [26], to three flavors and to higher order in $\delta_{I}$. The full calculations are in the Supplemental Material [27]. Here, we provide simplified expressions to LO in $\delta_{I}$ and taking $m_{s} \gg m_{d} \approx 2 m_{u}$. The ALP- $P$ kinetic and mass mixing cause the $P$ fields to pick up small admixtures of the physical ALP state and vice versa:

$$
\begin{aligned}
& P \approx P_{\mathrm{phy}}+\frac{f_{\pi}}{f_{a}}\langle\boldsymbol{a} \boldsymbol{P}\rangle a_{\mathrm{phy}}, \\
& a \approx a_{\mathrm{phy}}-\frac{f_{\pi}}{f_{a}} \sum_{P}\langle\boldsymbol{a} \boldsymbol{P}\rangle P_{\mathrm{phy}} .
\end{aligned}
$$

Therefore, the ALP $U(3)$ matrix is

$\boldsymbol{a}=\left\langle\boldsymbol{a} \boldsymbol{\pi}^{0}\right\rangle \boldsymbol{\pi}^{0}+\langle\boldsymbol{a} \boldsymbol{\eta}\rangle \boldsymbol{\eta}+\left\langle\boldsymbol{a} \boldsymbol{\eta}^{\prime}\right\rangle \boldsymbol{\eta}^{\prime} \quad$ for $m_{a} \lesssim 1 \mathrm{GeV}$,

where the ALP- $P$ mixing factors are

$$
\begin{aligned}
\left\langle\boldsymbol{a} \boldsymbol{\pi}^{0}\right\rangle & \approx \mathcal{N}_{\pi^{0}} \frac{\delta_{I} m_{a}^{2}}{m_{a}^{2}-m_{\pi}^{2}}, \quad\langle\boldsymbol{a} \boldsymbol{\eta}\rangle \approx \mathcal{N}_{\eta}\left[\frac{m_{a}^{2}-m_{\pi^{0}}^{2} / 2}{m_{a}^{2}-m_{\eta}^{2}}\right], \\
\left\langle\boldsymbol{a} \boldsymbol{\eta}^{\prime}\right\rangle & \approx \mathcal{N}_{\eta^{\prime}}\left[\frac{m_{a}^{2}-2 m_{\pi^{0}}^{2}}{m_{a}^{2}-m_{\eta^{\prime}}^{2}}\right],
\end{aligned}
$$

and $\mathcal{N}_{\pi^{0}, \eta, \eta^{\prime}}=\frac{1}{2}, 1 / \sqrt{6}, 1 / 2 \sqrt{3}$ are the $\boldsymbol{P}$ normalization factors. At high masses, the $U(3)$ symmetry is expected to be restored; thus the ALP $U(3)$ representation should be

$$
\mathcal{C}_{u} \approx \mathcal{C}_{d} \approx \mathcal{C}_{s} \approx 1 \quad \text { for } m_{a} \gg \Lambda_{\mathrm{QCD}} .
$$

The $\mathcal{C}_{q}$ values obtained from Eq. (11) are close to unity near $1 \mathrm{GeV}$; therefore, we interpolate between the low-mass and high-mass regions by setting each $\mathcal{C}_{q}$ element to unity once it intersects unity above $m_{\eta^{\prime}}$ (see Fig. 1).

When $m_{a}$ is in the nonperturbative regime of QCD, this $U(3)$-based representation is the most natural one, and can

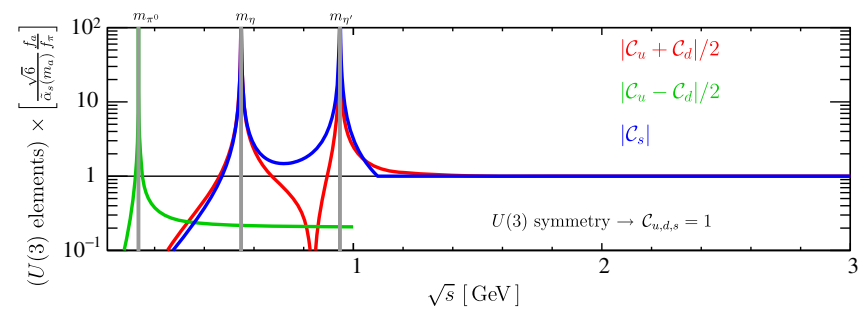

FIG. 1. ALP $U(3)$ representation. Since isospin-violating decays are small above $m_{\eta^{\prime}}$, where the isospin-violating component is highly uncertain, we ignore such decays for $m_{a}>m_{\eta^{\prime}}$. 
be used to calculate the production and decay rates of ALPs. Before moving onto such calculations, we stress that for $0.5 \lesssim m_{a} \lesssim 2 \mathrm{GeV}$ there are $\mathcal{O}(1)$ uncertainties on $\boldsymbol{a}$. Many LO $\chi$ PT predictions require $\mathcal{O}(1)$ corrections even for $\eta$ decays (see, e.g., Ref. [33]). Furthermore, while parton-hadron duality is roughly valid above $1 \mathrm{GeV}$ for vector currents [34], not enough is known about $\eta^{*}$ states to assert that this holds to better than $\mathcal{O}(1)$ for ALPs. While the precision of $\boldsymbol{a}$ could be improved, adding direct quark couplings to the ALP model also induces $\mathcal{O}(1)$ changes in $\boldsymbol{a}$. Therefore, a more natural approach is to adopt $\mathcal{C}_{u}, \mathcal{C}_{d}$, and $\mathcal{C}_{s}$ as effective ALP parameters, with the goal of experimentally exploring all $\mathcal{O}(1)$ deviations from the pure ALP-gluon model.

The interactions of pseudoscalar mesons are well described at low energies by the hidden local symmetries framework of vector meson dominance (VMD) [35,36]. Because of ALP-pseudoscalar mixing, which generates the ALP $U(3)$ representation, we can also employ VMD to study ALP interactions. However, since VMD only includes ground-state mesons, the effective theory breaks down once $m_{a} \gtrsim m_{\eta^{*}} \approx 1.5 \mathrm{GeV}$. Reference [34] showed how $e^{+} e^{-} \rightarrow V^{(*)}$ data can be used to predict the hadronic decay rates of any vector particle. While no high-purity source of $P^{(*)}$ currents exists, with minimal assumptions we can also use $e^{+} e^{-}$data to extend VMD-based pseudoscalar predictions up to $3 \mathrm{GeV}$.

We begin by considering an interaction vertex with two vectors and one pseudoscalar $(V V P)$. The amplitude for the process $V_{1}\left(p_{1}\right) \rightarrow V_{2}\left(p_{2}\right) P(q)$ must be of the form

$$
\begin{aligned}
\mathcal{A}_{V_{1} \rightarrow V_{2} P}= & \epsilon_{\mu \nu \alpha \beta} \epsilon_{1}^{\mu} \epsilon_{2}^{* \nu} p_{1}^{\alpha} p_{2}^{\beta} \mathcal{F}\left(p_{1}^{2}, p_{2}^{2}, q^{2}\right) \\
& \times \frac{3 g^{2}}{4 \pi^{2} f_{\pi}}\left\langle\boldsymbol{V}_{\mathbf{1}} \boldsymbol{V}_{\mathbf{2}} \boldsymbol{P}\right\rangle,
\end{aligned}
$$

since this is the only valid Lorentz structure. The unknown function $\mathcal{F}$ should satisfy

$\mathcal{F}\left(p_{1}^{2}, p_{2}^{2}, q^{2}\right)=\left\{\begin{array}{ll}\approx 1 & \text { for } m_{1} \ll m_{V_{1}^{*}}(\mathrm{VMD}) \\ \propto \frac{1}{m_{1}^{4}} & \text { for } m_{1} \gg \Lambda_{\mathrm{QCD}}(\mathrm{PQCD})\end{array}\right.$,

where $m_{1}^{2}=p_{1}^{2}$ and $m_{V_{1}^{*}}$ denotes the pole mass of the first excited vector meson with the same $U(3)$ representation as $V_{1}$. The PQCD power-counting rule is $\mathcal{A} \propto m_{1}^{4-n}$, where $n$ is the number of partons involved in the vertex ( 6 for $V V P$ ) [37]. Since for $m_{1} \lesssim m_{V_{1}^{*}} \mathcal{F}$ is approximately independent of the ground-state meson masses, we make the ansatz

$$
\mathcal{F}\left(p_{1}^{2}, p_{2}^{2}, q^{2}\right) \rightarrow \mathcal{F}\left(m_{1}\right),
$$

which relies on $\mathcal{F}$ being controlled by the heaviest dynamical scale, $m_{1}$ here, when all other masses are for ground-state mesons. As shown in Ref. [34], treating $e^{+} e^{-} \rightarrow q \bar{q}$ production as the sum of currents with $\rho$-like, $\omega$-like, and $\phi$-like $U(3)$ quantum numbers, rather than the sum of many $V^{*}$ resonances, provides a good description of the data for $m \equiv \sqrt{s} \gg m_{V^{*}}$. Therefore, the $\mathcal{F}$ function can be extracted from data using

$\mathcal{F}(m) \approx\left[\frac{3 m\left[\frac{\sigma_{e^{+} e^{-} \rightarrow f}(m)}{\sigma_{e^{+} e^{-} \rightarrow \mu^{+} \mu^{-}}(m)}\right]}{\Gamma_{V \rightarrow f}^{\mathrm{VMD}}(m)}\right]^{1 / 2} \times \begin{cases}\sqrt{\frac{2}{3}} & (\rho \text {-like }) \\ \sqrt{6} & (\omega \text {-like }) \\ \sqrt{3} & (\phi \text {-like })\end{cases}$

where $\Gamma_{V \rightarrow f}^{\mathrm{VMD}}(m)$ is the width obtained using VMD with $\mathcal{F}=1$.

Figure 2 shows that all available $e^{+} e^{-} \rightarrow V_{1} \rightarrow V_{2} P$ data are consistent with

$\mathcal{F}(m)= \begin{cases}1 & \text { for } m<1.4 \mathrm{GeV} \\ \text { interpolation } & \text { for } 1.4 \leq m \leq 2 \mathrm{GeV} \\ {\left[\frac{\beta_{\mathcal{F}}}{m}\right]^{4}} & \text { for } m>2 \mathrm{GeV}\end{cases}$

where $\beta_{\mathcal{F}}=1.4 \mathrm{GeV}$ is determined from the data. Furthermore, in the Supplemental Material [27] we show that all $e^{+} e^{-} \rightarrow V \rightarrow P P$ data $[38,39]$ are also consistent with Eq. (17), modulo the PQCD power-law scaling is $m^{-3}$ due to the dimensionality of the VMD-based VPP vertex. Since $\mathcal{F}$ is simply a smooth monotonic transition from VMD to PQCD, we expect this function to be approximately valid for any 3-meson vertex where only the decaying particle is not a ground-state meson (corrected for vertex dimensionality if needed). We will show below how to use Eq. (17) to extend VMD-based calculations up to $3 \mathrm{GeV}$, and validate our approach using known $\eta_{c}$ and $\eta^{*}$ decay branching fractions.

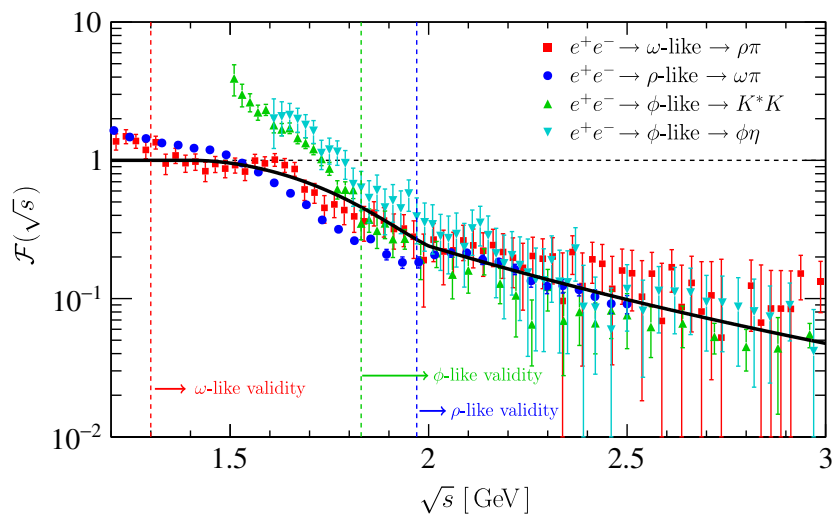

FIG. 2. $\mathcal{F}$ from Eq. (13) determined from $e^{+} e^{-}$data [40-42]. Since we ignore resonance contributions, each result is only valid at masses where narrow resonance contributions are small. We define these as ( $\omega$-like) above where the sizable $\omega-\phi$ interference effect in the $3 \pi$ final state becomes negligible, ( $\rho$-like) $m_{a} \gtrsim m_{\rho^{*}}+\Gamma_{\rho^{*}}$, and $\left(\phi\right.$-like) $m_{a} \gtrsim m_{\phi^{*}}+\Gamma_{\phi^{*}}$. 
TABLE I. Validation using $\eta_{c} \rightarrow V V$ decays: Our predictions are consistent with the PDG average of each experimental value $[44,45]$. Furthermore, we derive more precise experimental values by averaging the PDG $\eta_{c} \rightarrow V V$ results assuming $S U(3)$ symmetry in these decays [the $S U(3)$ column], and find that our predictions are consistent with these $S U(3)$-averaged experimental results to $\mathcal{O}(10 \%)$.

\begin{tabular}{lccc}
\hline \hline & This work & \multicolumn{2}{c}{ Experiment } \\
\cline { 2 - 4 } & $\mathrm{VMD} \times|\mathcal{F}(m)|^{2}$ & PDG & $S U(3)$ \\
\hline $\mathcal{B}\left(\eta_{c} \rightarrow \rho \rho\right)$ & $1.0 \%$ & $1.8 \pm 0.5 \%$ & $1.10 \pm 0.14 \%$ \\
$\mathcal{B}\left(\eta_{c} \rightarrow \omega \omega\right)$ & $0.40 \%$ & $0.20 \pm 0.10 \%$ & $0.44 \pm 0.06 \%$ \\
$\mathcal{B}\left(\eta_{c} \rightarrow \phi \phi\right)$ & $0.25 \%$ & $0.28 \pm 0.04 \%$ & $0.28 \pm 0.04 \%$ \\
$\mathcal{B}\left(\eta_{c} \rightarrow K^{*} \bar{K}^{*}\right)$ & $0.91 \%$ & $0.91 \pm 0.26 \%$ & $1.00 \pm 0.13 \%$ \\
\hline \hline
\end{tabular}

The amplitude for $P \rightarrow V_{1} V_{2}$ must have the same Lorentz structure as Eq. (13), and by crossing symmetry must share the same $\mathcal{F}$. Therefore, using the standard VMD framework-but inserting $\mathcal{F}\left(m_{P}\right)$-we can calculate $\Gamma_{a \rightarrow V V}\left(m_{a}\right)$ up to $\approx 3 \mathrm{GeV}$. These straightforward calculations follow directly from the standard VMD ones and are provided in the Supplemental Material [27]. Moreover, using the same framework we calculate $\Gamma_{\eta_{c} \rightarrow V V}$. Table I shows that our $\eta_{c} \rightarrow V V$ predictions are consistent with the experimental values to $\mathcal{O}(10 \%)$. Alternatively, $\Gamma_{\eta_{c} \rightarrow V V}$ can be calculated using PQCD; however, this approach underestimates the measurements [43] by $\mathcal{O}(10)$ even when including higher-twist effects (known as the $\eta_{c} \rightarrow V V$ puzzle). That our predictions for $\Gamma_{\eta_{c} \rightarrow V V}$ achieve $\mathcal{O}(10 \%)$ accuracy provides strong validation of the approach developed here.

Given any ALP $U(3)$ representation and the massdependent vertex scaling function Eq. (17), we can calculate exclusive hadronic ALP decay widths and its total hadronic width. Here we summarize our calculations for the representation shown in Fig. 1, while the details are provided in the Supplemental Material [27].

(1) $\Gamma_{a \rightarrow V V}$ : As discussed above, we calculate $a \rightarrow \rho \rho$, $a \rightarrow \omega \omega, a \rightarrow \phi \phi$, and $a \rightarrow K^{*} \bar{K}^{*}$ using our extendedVMD framework. Schematically, the $a \rightarrow V V$ and VMDbased $\eta^{\prime} \rightarrow V V$ rates are related via

$$
\Gamma_{a \rightarrow V V}=\left|\frac{f_{\pi}\langle\boldsymbol{a}\{\boldsymbol{V}, \boldsymbol{V}\}\rangle \mathcal{F}\left(m_{a}\right)}{f_{a}\left\langle\boldsymbol{\eta}^{\prime}\{\boldsymbol{V}, \boldsymbol{V}\}\right\rangle}\right|^{2} \Gamma_{\eta^{\prime} \rightarrow V V}^{m_{\eta^{\prime} \rightarrow m_{a}}} .
$$

Additionally, we calculate $\Gamma_{a \rightarrow \pi \pi \gamma}$ as $a \rightarrow \rho \rho$ followed by $\rho-\gamma$ mixing and $\rho \rightarrow \pi \pi$.

(2) $\Gamma_{a \rightarrow V P}$ : Since $a \rightarrow \rho \pi$ violates isospin and $a \rightarrow K^{*} K$ violates $S U(3)$ symmetry, these are subleading and difficult to calculate; thus we do not consider them. (Determining the $U(3)$-violating components of $\boldsymbol{a}$ and the kaon-loop contributions to isospin-violating final states would be tedious. None of these $U(3)$-violating modes are expected to be important at any masses.) Most other $a \rightarrow V P$ decays involving ground-state mesons violate $C$, so also are not considered.
(3) $\Gamma_{a \rightarrow \gamma \gamma}$ : The $a \rightarrow \gamma \gamma$ decay rate is given by

$$
\Gamma_{a \rightarrow \gamma \gamma}=\frac{\alpha_{\mathrm{EM}}^{2} m_{a}^{3}}{(4 \pi)^{3} f_{a}^{2}}\left|\mathcal{C}_{\gamma}^{\chi}+\mathcal{C}_{\gamma}^{\mathrm{VMD}}+\mathcal{C}_{\gamma}^{\mathrm{PQCD}}\right|^{2},
$$

where at low masses $\mathcal{C}_{\gamma}^{\chi} \approx 1$ is generated by the chiral transformation, while at high masses PQCD quark-loop contributions (at two-loop order) are important [26]. Calculated for the first time here from $a \rightarrow V V \rightarrow \gamma \gamma$ with $V-\gamma$ mixing,

$$
\begin{aligned}
\mathcal{C}_{\gamma}^{\mathrm{VMD}} & =-\mathcal{F}\left(m_{a}\right)\left[3\langle\boldsymbol{a} \boldsymbol{\rho} \boldsymbol{\rho}\rangle+\frac{1}{3}\langle\boldsymbol{a} \boldsymbol{\omega} \boldsymbol{\omega}\rangle+\frac{2}{3}\langle\boldsymbol{a} \phi \phi\rangle+2\langle\boldsymbol{a} \boldsymbol{\rho} \boldsymbol{\omega}\rangle\right] \\
& =-\mathcal{F}\left(m_{a}\right) \frac{2 \tilde{\alpha}_{s}\left(m_{a}\right)}{3 \sqrt{6}}\left(4 \mathcal{C}_{u}+\mathcal{C}_{d}+\mathcal{C}_{s}\right),
\end{aligned}
$$

is found to be the dominant contribution over most of the mass range considered. Nota bene, each contribution is turned on or off for $m_{a}$ values where it is either invalid or where double counting of contributions would occur.

(4) $\Gamma_{a \rightarrow 3 \pi}$ : We calculate these rates using the LO chiral Lagrangian, and add a data-derived $k$ factor to account for final-state-pion rescattering effects. We only consider these decays up to $m_{\eta^{\prime}}$, since at higher masses this $k$ factor is no longer reliable. We consider isospin-violating $a-\pi^{0}$ mixing, and our calculation is the first to consider $a-\eta^{(\prime)}$ mixing followed by $\eta^{(\prime)} \rightarrow 3 \pi$. We leave a detailed presentation to the Supplemental Material [27].

(5) $\Gamma_{a \rightarrow P P P}$ : The amplitudes for $a \rightarrow \eta^{(\prime)} \pi \pi$ and $a \rightarrow K \bar{K} \pi$ are dominated by scalar and tensor resonances. Specifically, for $a \rightarrow \eta^{(\prime)} \pi \pi$ we consider $a \rightarrow \sigma(\pi \pi) \eta^{(\prime)}, a \rightarrow f_{0}(\pi \pi) \eta^{(\prime)}$, $a \rightarrow a_{0}\left(\eta^{(\prime)} \pi\right) \pi, a \rightarrow f_{2}(\pi \pi) \eta^{(\prime)}$, and a contact term. For $a \rightarrow K \bar{K} \pi$ we consider $a \rightarrow S_{K \pi}(K \pi) K$, where the $K \pi S$ wave amplitude is taken from Ref. [46], and $a \rightarrow a_{0}(K K) \pi$. Schematically, the $a \rightarrow P P P$ and $\eta^{\prime} \rightarrow P P P$ amplitudes are related similarly to Eq. (18), e.g.,

$$
\mathcal{A}_{a \rightarrow f_{0}(\pi \pi) \eta}=\frac{f_{\pi}\left\langle\boldsymbol{a} \boldsymbol{\eta} \boldsymbol{f}_{\mathbf{0}}\right\rangle \mathcal{F}\left(m_{a}\right)}{f_{a}\left\langle\boldsymbol{\eta}^{\prime} \boldsymbol{\eta} \boldsymbol{f}_{\mathbf{0}}\right\rangle} \mathcal{A}_{\eta^{\prime} \rightarrow f_{0}(\pi \pi) \eta}^{m_{\eta^{\prime}} \rightarrow m_{a}} .
$$

All scalar resonance amplitudes are taken from the $\eta^{\prime} \rightarrow \eta \pi \pi$ model of Ref. [47], where they were determined by fitting all available data. We use a similar approach to derive the $f_{2}(1270)$ tensor-meson contribution in the Supplemental Material [27]. Unlike above, we cannot obtain the $\mathcal{F}$ functions for these vertices directly from data. Given that the dimensionality of each of these vertices is the same as that of $V V P$, we also use Eq. (17) here. This universality assumption is validated by the fact that we accurately predict both $\mathcal{B}\left(\eta_{c} \rightarrow \eta \pi \pi\right)$ and $\mathcal{B}(\eta(1760) \rightarrow \gamma \gamma) \times \mathcal{B}(\eta(1760) \rightarrow$ $\left.\eta^{\prime} \pi \pi\right)$ to $\approx 20 \%$, and $\mathcal{B}\left(\eta_{c} \rightarrow K \bar{K} \pi\right)$ to $\approx 10 \%[44,48]$. Given that $a \rightarrow \eta \pi \pi$ or $a \rightarrow K \bar{K} \pi$ has the largest branching fraction for $m_{a} \gtrsim 1 \mathrm{GeV}$, the lack of more stringent data-driven constraints here is the weakest component of our calculations, 


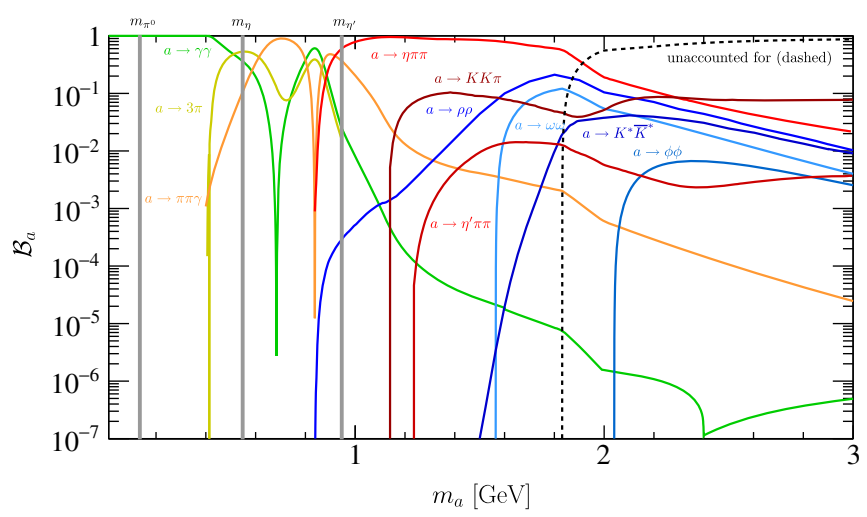

FIG. 3. ALP decay branching fractions to all final states considered; decay widths are given in the Supplemental Material [27].

though these data-driven tests suggest that the uncertainties are small. (These predictions could be improved with a better experimental understanding of the excited $\eta^{*}$ states).

(6) $\Gamma_{a \rightarrow g g}$ : The next-to-LO PQCD calculation of Eq. (4) derived in Ref. [26] is adopted here.

(7) $\Gamma_{a}$ (total hadronic width): We take $\Gamma_{a}=\Gamma_{a \rightarrow g g}$ for $m_{a} \gtrsim 1.84 \mathrm{GeV}$, while for lower masses, the sum of all exclusive modes is used for $\Gamma_{a}$. At $m_{a} \simeq 1.84 \mathrm{GeV}$ we find $\Gamma_{a \rightarrow g g} \approx \sum_{\mathrm{i}=\mathrm{exc}} \Gamma_{i}$.

The decay branching fractions are summarized in Fig. 3. The unaccounted for branching fraction is also shown, and is substantial for $m_{a} \gtrsim 2 \mathrm{GeV}$. This includes decays such as $a \rightarrow A A$, i.e., two axial-vector mesons, which should be comparable to $a \rightarrow V V$ above about $2.5 \mathrm{GeV}$, and many decay paths that involve excited resonances, rescatterings, etc. For example $\mathcal{B}\left(\eta_{c} \rightarrow 6 \pi\right) \approx 20 \%$ so we expect ALP decays to many-body final states to be at about the same rate. We stress that unaccounted for decay modes should only be important for ALP masses where $\Gamma_{a} \approx \Gamma_{a \rightarrow g g}$; therefore, our predictions for the total hadronic widthand the ALP lifetime-should not be affected by unaccounted for decays.

When evaluating the constraints on this model, we focus on the $m_{\pi}<m_{a}<3 \mathrm{GeV}$ region, where our work has the biggest impact. Constraints where $f_{a} \lesssim 3 f_{\pi}$ are omitted, e.g., bounds from radiative $J / \psi$ decays, since we assumed $f_{\pi} \ll f_{a}$ when deriving $\boldsymbol{a}$. Details on all calculations are provided in the Supplemental Material [27], while in Fig. 4 and below we summarize the constraints.

(1) We recast existing limits on the $a \gamma \gamma$ vertex from LEP [20,49] and beam-dump experiments [50-52] using our $\mathcal{B}(a \rightarrow \gamma \gamma)$ result and our $a \rightarrow \gamma \gamma$ calculation to relate the $a \gamma \gamma$ interaction strength to $f_{a}$. In Ref. [53], we derive new constraints using $\gamma p \rightarrow p a(\gamma \gamma)$ data from GluEX [54].

(2) We derive new constraints from $\phi \rightarrow \gamma a\left(\pi \pi \gamma, \eta \pi^{0} \pi^{0}\right)$ and $\eta^{\prime} \rightarrow \pi^{+} \pi^{-} a\left(\pi^{+} \pi^{-} \pi^{0}\right)$. We are not aware of any bump hunts here, and instead assume that the entire known branching fractions to these final states [44] are due to

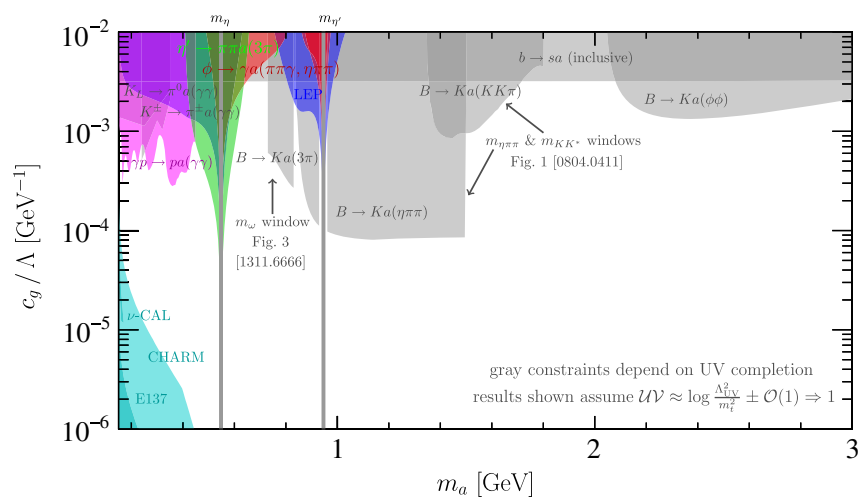

FIG. 4. Constraints on the ALP-gluon coupling.

ALPs. Clearly dedicated searches would be much more sensitive.

(3) We derive new constraints from $b \rightarrow s a$ penguin decays. At one loop, the agg vertex generates an axial-vector att coupling [26] resulting in enhanced rates for $B \rightarrow K^{(*)} a$ decays [55-58]. The loop contains a UV-dependent factor [59] schematically given by $\approx\left[\log \Lambda_{\mathrm{UV}}^{2} / m_{t}^{2} \pm \mathcal{O}(1)\right]$, which we take to be unity [corresponding to an $\mathcal{O}(\mathrm{TeV}) \mathrm{UV}$ scale]. This induces $\mathcal{O}(1)$ arbitrariness on the following constraints:

(i) The published $m_{\eta \pi \pi}$ spectrum of Ref. [60] is used to constrain $\mathcal{B}\left(B^{ \pm} \rightarrow K^{ \pm} a\right) \times \mathcal{B}\left(a \rightarrow \eta \pi^{+} \pi^{-}\right)$for $m_{a}<1.5 \mathrm{GeV}$, excluding the $\eta^{\prime}$ peak region.

(ii) The published $m_{K^{*} K}$ spectrum of Ref. [60] is used to constrain $\mathcal{B}\left(B^{ \pm} \rightarrow K^{ \pm} a\right) \times \mathcal{B}\left(a \rightarrow K^{ \pm} K_{S} \pi^{\mp}\right)$ for $0.85<$ $m_{K \pi}<0.95 \mathrm{GeV}$ and $m_{a}<1.8 \mathrm{GeV}$.

(iii) The known value of $\mathcal{B}\left(B^{0} \rightarrow K^{0} \phi \phi\right)$ [61] is used to constrain $\mathcal{B}\left(B^{0} \rightarrow K^{0} a\right) \times \mathcal{B}(a \rightarrow \phi \phi)$ assuming the entire decay rate is due to ALPs.

(iv) The known value of $\mathcal{B}\left(B^{ \pm} \rightarrow K^{ \pm} \omega(3 \pi)\right)$ is used to constrain $\mathcal{B}\left(B^{ \pm} \rightarrow K^{ \pm} a\right) \times \mathcal{B}\left(a \rightarrow \pi^{+} \pi^{-} \pi^{0}\right)$ for $0.73<$ $m_{a}<0.83 \mathrm{GeV}$, which is the $3 \pi$ mass window shown in Ref. [62], assuming the entire decay rate is due to ALPs.

(v) Since the ALPs considered here are not massive enough to decay into charm hadrons, the observed inclusive $b \rightarrow c$ branching fraction [44] is used to place an upper limit on the inclusive $b \rightarrow s a$ rate of $\mathcal{B}(b \rightarrow s a)<[1-\mathcal{B}(b \rightarrow c)]$.

(4) Similarly, we recast existing limits on ALP $-W / Z$ couplings from Ref. [19] using the $s \rightarrow d$ penguin decays $K^{ \pm} \rightarrow \pi^{ \pm} \gamma \gamma$ [63] and $K_{L} \rightarrow \pi^{0} \gamma \gamma$ [64] and the same UVcompletion assumptions.

Over much of the considered mass range the constraints on $\Lambda$ are below a TeV. We stress that many of these constraints would be much stronger if dedicated searches were performed, e.g., searches for $B \rightarrow K^{(*)} a$ with $a \rightarrow \gamma \gamma, 3 \pi, \eta \pi \pi, K \bar{K} \pi, \rho \rho$, etc. would be incredibly powerful probes of QCD-scale ALPs - and could be performed with data already collected by LHCb.

In summary, we presented a novel data-driven method for determining the hadronic interaction strengths of ALPs 
with QCD-scale masses. Our method makes it possible to calculate the hadronic production and decay rates of ALPs, along with many of the largest ALP decay branching fractions to exclusive final states. To illustrate the impact on QCD-scale ALP phenomenology, we considered the scenario where the ALP-gluon coupling is dominant over the ALP coupling to photons, electroweak bosons, and all fermions, but emphasized that our method is easily generalized to any set of ALP couplings to SM particles. We showed that the constraints on this type of ALP are weak, though we also highlighted some promising searches that could provide improved sensitivity to QCD-scale ALPs, e.g., at LHCb. Finally, our work determined the relationship between the ALP lifetime and its gluonic coupling, which is vital for studying the sensitivity of long-lived particle experiments [65].

We thank Jesse Thaler for providing feedback on the early stages of this work and for providing comments on this Letter, along with Will Detmold, Michele Papucci, and Maxim Pospelov for providing feedback on this Letter, and David Curtin, Phil Ilten, and Jure Zupan for useful discussions. Y. S. and M. W. performed part of this work at the Aspen Center for Physics, which is supported by U.S. National Science Foundation Grant No. PHY-1607611. This work was supported by: Y. S. was supported by the Office of High Energy Physics of the U.S. Department of Energy under Grant Contract No. DE-SC00015476; and M. W. was supported by the U.S. National Science Foundation under Contract No. PHY-1607225.

*daniel.aloni@weizmann.ac.il

†yotam.soreq@cern.ch

*mwill@mit.edu

[1] R. Essig et al., Working Group Report: New light weakly coupled particles, arXiv:1311.0029.

[2] D. J. E. Marsh, Axion cosmology, Phys. Rep. 643, 1 (2016).

[3] P. W. Graham, I. G. Irastorza, S. K. Lamoreaux, A. Lindner, and K. A. van Bibber, Experimental searches for the axion and axion-like particles, Annu. Rev. Nucl. Part. Sci. 65, 485 (2015).

[4] I. G. Irastorza and J. Redondo, New experimental approaches in the search for axion-like particles, Prog. Part. Nucl. Phys. 102, 89 (2018).

[5] R. D. Peccei and H.R. Quinn, $C P$ Conservation in the Presence of Instantons, Phys. Rev. Lett. 38, 1440 (1977).

[6] R. D. Peccei and H. R. Quinn, Constraints imposed by $C P$ conservation in the presence of instantons, Phys. Rev. D 16, 1791 (1977).

[7] S. Weinberg, A New Light Boson? Phys. Rev. Lett. 40, 223 (1978).

[8] F. Wilczek, Problem of Strong $P$ and $T$ Invariance in the Presence of Instantons, Phys. Rev. Lett. 40, 279 (1978).

[9] P. W. Graham, D. E. Kaplan, and S. Rajendran, Cosmological Relaxation of the Electroweak Scale, Phys. Rev. Lett. 115, 221801 (2015).
[10] D. Chang, W.-F. Chang, C.-H. Chou, and W.-Y. Keung, Large two loop contributions to g-2 from a generic pseudoscalar boson, Phys. Rev. D 63, 091301(R) (2001).

[11] Y. Nomura and J. Thaler, Dark Matter through the axion portal, Phys. Rev. D 79, 075008 (2009).

[12] M. Freytsis and Z. Ligeti, On dark matter models with uniquely spin-dependent detection possibilities, Phys. Rev. D 83, 115009 (2011).

[13] M. J. Dolan, F. Kahlhoefer, C. McCabe, and K. SchmidtHoberg, A taste of dark matter: Flavour constraints on pseudoscalar mediators, J. High Energy Phys. 03 (2015) 171; Erratum, J. High Energy Phys. 07 (2015) 103(E).

[14] Y. Hochberg, E. Kuflik, R. Mcgehee, H. Murayama, and K. Schutz, SIMPs through the axion portal, Phys. Rev. D 98, 115031 (2018).

[15] D. S. M. Alves and N. Weiner, A viable QCD axion in the MeV mass range, J. High Energy Phys. 07 (2018) 092.

[16] W. J. Marciano, A. Masiero, P. Paradisi, and M. Passera, Contributions of axionlike particles to lepton dipole moments, Phys. Rev. D 94, 115033 (2016).

[17] J. Jaeckel and M. Spannowsky, Probing MeV to $90 \mathrm{GeV}$ axion-like particles with LEP and LHC, Phys. Lett. B 753, 482 (2016).

[18] B. Dobrich, J. Jaeckel, F. Kahlhoefer, A. Ringwald, and K. Schmidt-Hoberg, ALPtraum: ALP production in proton beam dump experiments, J. High Energy Phys. 02 (2016) 018.

[19] E. Izaguirre, T. Lin, and B. Shuve, Searching for Axionlike Particles in Flavor-Changing Neutral Current Processes, Phys. Rev. Lett. 118, 111802 (2017).

[20] S. Knapen, T. Lin, H. K. Lou, and T. Melia, Searching for Axionlike Particles with Ultraperipheral Heavy-Ion Collisions, Phys. Rev. Lett. 118, 171801 (2017).

[21] A. V. Artamonov et al. (BNL-E949 Collaboration), Study of the decay $K^{+} \rightarrow \pi^{+} \nu \bar{\nu}$ in the momentum region $140<$ $P_{\pi}<199 \mathrm{MeV} / \mathrm{c}$, Phys. Rev. D 79, 092004 (2009).

[22] H. Fukuda, K. Harigaya, M. Ibe, and T. T. Yanagida, Model of visible QCD axion, Phys. Rev. D 92, 015021 (2015).

[23] M. Bauer, M. Heiles, M. Neubert, and A. Thamm, Axionlike particles at future colliders, Eur. Phys. J. C 79, 74 (2019).

[24] A. Mariotti, D. Redigolo, F. Sala, and K. Tobioka, New LHC bound on low-mass diphoton resonances, Phys. Lett. B 783, 13 (2018).

[25] X. Cid Vidal, A. Mariotti, D. Redigolo, F. Sala, and K. Tobioka, New axion searches at flavor factories, J. High Energy Phys. 01 (2019) 113.

[26] M. Bauer, M. Neubert, and A. Thamm, Collider probes of axion-like particles, J. High Energy Phys. 12 (2017) 044.

[27] See Supplemental Material at http://link.aps.org/ supplemental/10.1103/PhysRevLett.123.031803 for technical details about our calculations and more detailed cross checks of our results.

[28] B. A. Dobrescu, G. L. Landsberg, and K. T. Matchev, Higgs boson decays to $C P$ odd scalars at the tevatron and beyond, Phys. Rev. D 63, 075003 (2001).

[29] M. Spira, A. Djouadi, D. Graudenz, and P. M. Zerwas, Higgs boson production at the LHC, Nucl. Phys. B453, 17 (1995). 
[30] H. Georgi, D. B. Kaplan, and L. Randall, Manifesting the invisible axion at low-energies, Phys. Lett. 169B, 73 (1986).

[31] W. A. Bardeen, R. D. Peccei, and T. Yanagida, Constraints on variant axion models, Nucl. Phys. B279, 401 (1987).

[32] L. M. Krauss and M. B. Wise, Constraints on shortlived axions from the decay $\pi^{+} \rightarrow e^{+} e^{-} e^{+}$neutrino, Phys. Lett. B 176, 483 (1986).

[33] J. Bijnens and K. Ghorbani, $\eta \rightarrow 3 \pi$ at two loops in chiral perturbation theory, J. High Energy Phys. 11 (2007) 030.

[34] P. Ilten, Y. Soreq, M. Williams, and W. Xue, Serendipity in dark photon searches, J. High Energy Phys. 06 (2018) 004.

[35] T. Fujiwara, T. Kugo, H. Terao, S. Uehara, and K. Yamawaki, Nonabelian anomaly and vector mesons as dynamical gauge bosons of hidden local symmetries, Prog. Theor. Phys. 73, 926 (1985).

[36] J. J. Sakurai, Theory of strong interactions, Ann. Phys. (N.Y.) 11, 1 (1960).

[37] G. P. Lepage and S. J. Brodsky, Exclusive processes in perturbative quantum chromodynamics, Phys. Rev. D 22, 2157 (1980).

[38] J. P. Lees et al. (BABAR Collaboration), Precise measurement of the $e^{+} e^{-} \rightarrow \pi^{+} \pi^{-}(\gamma)$ cross section with the initialstate radiation method at BABAR, Phys. Rev. D 86, 032013 (2012).

[39] J. P. Lees et al. (BABAR Collaboration), Precision measurement of the $e^{+} e^{-} \rightarrow K^{+} K^{-}(\gamma)$ cross section with the initial-state radiation method at BABAR, Phys. Rev. D 88, 032013 (2013).

[40] J. P. Lees et al. (BABAR Collaboration), Measurement of the $e^{+} e^{-} \rightarrow \pi^{+} \pi^{-} \pi^{0} \pi^{0}$ cross section using initial-state radiation at BABAR, Phys. Rev. D 96, 092009 (2017).

[41] B. Aubert et al. (BABAR Collaboration), Measurements of $e^{+} e^{-} \rightarrow K^{+} K^{-} \eta, K^{+} K^{-} \pi^{0}$ and $K_{s}^{0} K^{ \pm} \pi^{\mp}$ cross sections using initial state radiation events, Phys. Rev. D 77, 092002 (2008).

[42] B. Aubert et al. (BABAR Collaboration), Study of $e^{+} e^{-} \rightarrow$ $\pi^{+} \pi^{-} \pi^{0}$ process using initial state radiation with BABAR, Phys. Rev. D 70, 072004 (2004).

[43] P. Sun, G. Hao, and C.-F. Qiao, Pseudoscalar quarkonium exclusive decays to vector meson pair, Phys. Lett. B 702, 49 (2011).

[44] M. Tanabashi et al. (Particle Data Group Collaboration), Review of particle physics, Phys. Rev. D 98, 030001 (2018).

[45] Z. Q. Liu et al. (Belle Collaboration), Observation of New Resonant Structures in $\gamma \gamma \rightarrow \omega \phi, \phi \phi$ and $\omega \omega$, Phys. Rev. Lett. 108, 232001 (2012).

[46] J. P. Lees et al. (BABAR Collaboration), Measurement of the $\mathrm{I}=1 / 2 K \pi \mathcal{S}$-wave amplitude from Dalitz plot analyses of $\eta_{c} \rightarrow K \bar{K} \pi$ in two-photon interactions, Phys. Rev. D 93, 012005 (2016).

[47] A. H. Fariborz and J. Schechter, $\eta^{\prime} \rightarrow \eta \pi \pi$ decay as a probe of a possible lowest lying scalar nonet, Phys. Rev. D 60 , 034002 (1999).

[48] Q. N. Xu et al. (Belle Collaboration), Measurement of $\eta_{c}(1 S)$, eta $(2 S)$ and non-resonant $\eta^{\prime} \pi^{+} \pi^{-}$production via two-photon collisions, Phys. Rev. D 98, 072001 (2018).
[49] G. Abbiendi et al. (OPAL Collaboration), Multiphoton production in $\mathrm{e}+\mathrm{e}-$ collisions at $\mathrm{s} * *(1 / 2)=181-\mathrm{GeV}$ to 209-GeV, Eur. Phys. J. C 26, 331 (2003).

[50] J. D. Bjorken, S. Ecklund, W. R. Nelson, A. Abashian, C. Church, B. Lu, L. W. Mo, T. A. Nunamaker, and P. Rassmann, Search for neutral metastable penetrating particles produced in the SLAC beam dump, Phys. Rev. D 38, 3375 (1988).

[51] J. Blümlein et al., Limits on neutral light scalar and pseudoscalar particles in a proton beam dump experiment, Z. Phys. C 51, 341 (1991).

[52] A. Ariga et al. (FASER Collaboration), FASERs physics reach for long-lived particles, Phys. Rev. D 99, 095011 (2019).

[53] D. Aloni, C. Fanelli, Y. Soreq, and M. Williams, Photoproduction of axion-like particles, arXiv:1903.03586.

[54] H. Al Ghoul et al. (GlueX Collaboration), Measurement of the beam asymmetry $\Sigma$ for $\pi^{0}$ and $\eta$ photoproduction on the proton at $E_{\gamma}=9 \mathrm{GeV}$, Phys. Rev. C 95, 042201 (2017).

[55] B. Batell, M. Pospelov, and A. Ritz, Multi-lepton signatures of a hidden sector in rare B decays, Phys. Rev. D 83, 054005 (2011).

[56] G. Hiller, B physics signals of the lightest $C P$ odd Higgs in the NMSSM at large tan beta, Phys. Rev. D 70, 034018 (2004).

[57] C. Bobeth, T. Ewerth, F. Kruger, and J. Urban, Analysis of neutral Higgs boson contributions to the decays $\bar{B}(s) \rightarrow \ell^{+} \ell^{-}$and $\bar{B} \rightarrow K \ell^{+} \ell^{-}$, Phys. Rev. D 64, 074014 (2001).

[58] K. Choi, S. H. Im, C. B. Park, and S. Yun, Minimal flavor violation with axion-like particles, J. High Energy Phys. 11 (2017) 070,

[59] M. Freytsis, Z. Ligeti, and J. Thaler, Constraining the axion portal with $B \rightarrow K l^{+} l^{-}$, Phys. Rev. D 81, 034001 (2010).

[60] B. Aubert et al. (BABAR Collaboration), Study of B Meson Decays with Excited Eta and Eta-Prime Mesons, Phys. Rev. Lett. 101, 091801 (2008).

[61] J. P. Lees et al. (BABAR Collaboration), Measurements of branching fractions and $C P$ asymmetries and studies of angular distributions for $B \rightarrow \phi \phi K$ decays, Phys. Rev. D 84, 012001 (2011).

[62] V. Chobanova et al. (Belle Collaboration), Measurement of branching fractions and $C P$ violation parameters in $B \rightarrow \omega K$ decays with first evidence of $C P$ violation in $B^{0} \rightarrow \omega K_{S}^{0}$, Phys. Rev. D 90, 012002 (2014).

[63] C. Lazzeroni et al. (NA62 Collaboration), Study of the $K^{ \pm} \rightarrow \pi^{ \pm} \gamma \gamma$ decay by the NA62 experiment, Phys. Lett. B 732, 65 (2014).

[64] E. Abouzaid et al. (KTeV Collaboration), Final results from the $\mathrm{KTeV}$ experiment on the decay $K_{L} \rightarrow \pi^{0} \gamma \gamma$, Phys. Rev. D 77, 112004 (2008).

[65] J. Beacham et al., Physics beyond colliders at CERN: Beyond the Standard Model Working Group Report, arXiv:1901.09966. 